\title{
Translating New Lung Cancer Screening Guidelines into Practice: The Experience of One Community Hospital
}

\author{
Christy J. W. Ledford, PhD, Breanna L. Gawrys, DO, Jessica L. Wall, MD, \\ Patrick D. Saas, MD, and Dean A. Seebusen, MD, MPH
}

Introduction: In December 2013 the US Preventive Services Task Force issued a recommendation for lung cancer screening with annual low-dose computed tomography (LDCT). As screening guidelines emerge and change, this creates an environment for studying the translation of these guidelines into practice. This study assessed how these guidelines were implemented in a community hospital setting and the resulting radiologic findings.

Methods: This observational study examined the radiologic outcomes of LDCT lung cancer screening guidelines and the resulting notification.

Results: During the first year after publication of the guidelines, 94 screening LDCT scans were ordered. Of these, $21(22.3 \%)$ did not meet the criteria outlined by the US Preventive Services Task Force. Among the 72 cases that did met published criteria, $65.3 \%$ of scans detected nodules, and among the remaining $35.6 \%$, half had another clinically significant finding.

Discussion: This study shows that new lung cancer screening guidelines, as implemented at a community hospital, resulted in radiologic findings that required follow-up in more than half of patients. Clinicians must be aware of these potential incidental findings when talking to patients about the decision to order screenings. (J Am Board Fam Med 2016;29:152-155.)

Keywords: Cancer Screening, Decision Making, Lung Cancer, Patient-centered Care

Lung cancer is the third most common cancer in the United States and the most common cancerrelated cause of death. ${ }^{1}$ In December 2013 the US Preventive Services Task Force (USPSTF) issued a "B" recommendation for lung cancer screening

This article was externally peer reviewed.

Submitted 9 April 2015; revised 6 August 2015; accepted 12 August 2015.

From the Department of Family Medicine, Uniformed Services University of the Health Sciences, Bethesda, MD (CJWL); the National Capital Consortium Family Medicine Residency Program, Fort Belvoir Community Hospital, Fort Belvoir, VA (BLG, JLW, PDS); and the Department of Family and Community Medicine, Dwight D. Eisenhower Army Medical Center, Augusta, GA (DAS).

Funding: This study was funded by an intramural research grant from Uniformed Services University of the Health Sciences (grant no. R081 2921).

Conflict of interest: none declared.

Disclaimer: The opinions and assertions contained herein are the private views of the authors and are not to be construed as official or as reflecting the views of the US Air Force, US Army, US Navy, Uniformed Services University of the Health Sciences, or the Department of Defense at large. with annual low-dose computed tomography (LDCT), ${ }^{2}$ based largely on the results of the $\mathrm{Na}$ tional Lung Screening Trial (NLST). ${ }^{3}$ There has been considerable debate regarding the true magnitude of the benefits and costs of such a strategy when widely implemented. ${ }^{4}$

The population target of the USPSTF resembles the inclusion criteria of the NLST, a large and well-conducted study. Still, the study population and conditions did not exactly match patients encountered in everyday practice. NLST participants were younger, more educated, and more likely to be current smokers than the general population. ${ }^{5}$ From 2004 to 2006, more than half the cases of lung cancer occurred in patients $\geq 70$ years old ${ }^{6}$;

Corresponding author: Christy J. W. Ledford, PhD, Department of Family Medicine, Uniformed Services University of the Health Sciences, FAM A1040, 4301 Jones Bridge Rd, Bethesda, MD 20814 (E-mail: christian.ledford@usuhs. edu). 
however, $<9 \%$ of NLST participants were age 70 or older. ${ }^{3}$ Therefore, patients screened in the general population will likely be older and have more comorbidities than in the NLST population. Participants in the NLST received annual LDCT screening for 3 years in academic health centers and were then followed clinically, but USPSTF extended the annual recommendation, in both timeline and context, to $>3$ years and beyond the context of academic health centers. Adding annual scans will pick up more cancers but will likely also cause additional false positives.

As screening guidelines emerge and change, it is vital to study the translation of guidelines into practice. This study assessed how these new guidelines were implemented in a community hospital setting and the resulting radiologic findings.

\section{Methods}

This observational study examined the radiologic outcomes of LDCT lung cancer screening guidelines in first year after publication by the USPSTF. Following exemption by the institutional review board, electronic medical records of all patients scheduled to receive an LDCT screening study in the radiology department of a community hospital were reviewed. To be included in data analysis, the LDCT must have been ordered for lung cancer screening, the patient must have been between 55 and 79 years old, and the patient must have had a documented smoking history ( $\geq 30$ pack-years) with recent smoking behavior (smoked within 15 years) (Figure 1).

Investigators reviewed the patient record for the ordering provider, determining whether the ordering provider was the primary care physician as assigned in the patient-centered medical home, a primary care provider was not assigned, or the primary care provider was a specialist. Investigators also recorded LDCT findings, orders for additional imaging studies (additional recommended before the next year's annual screening), and resulting diagnoses. For data analysis, scan results were coded according to the Fleischner Society recommendations for follow-up and management of nodules detected through computed tomography. ${ }^{7}$ This coding scheme ranged from 0 (no findings) to 4 (nodules $>8 \mathrm{~mm}$ ).

After this initial coding, a second coding scheme was applied to data to further elaborate findings
Figure 1. Flow diagram for inclusion of cases. CT, computed tomography; LDCT, low-dose computed tomography; USPSTF, US Preventive Services Task Force.

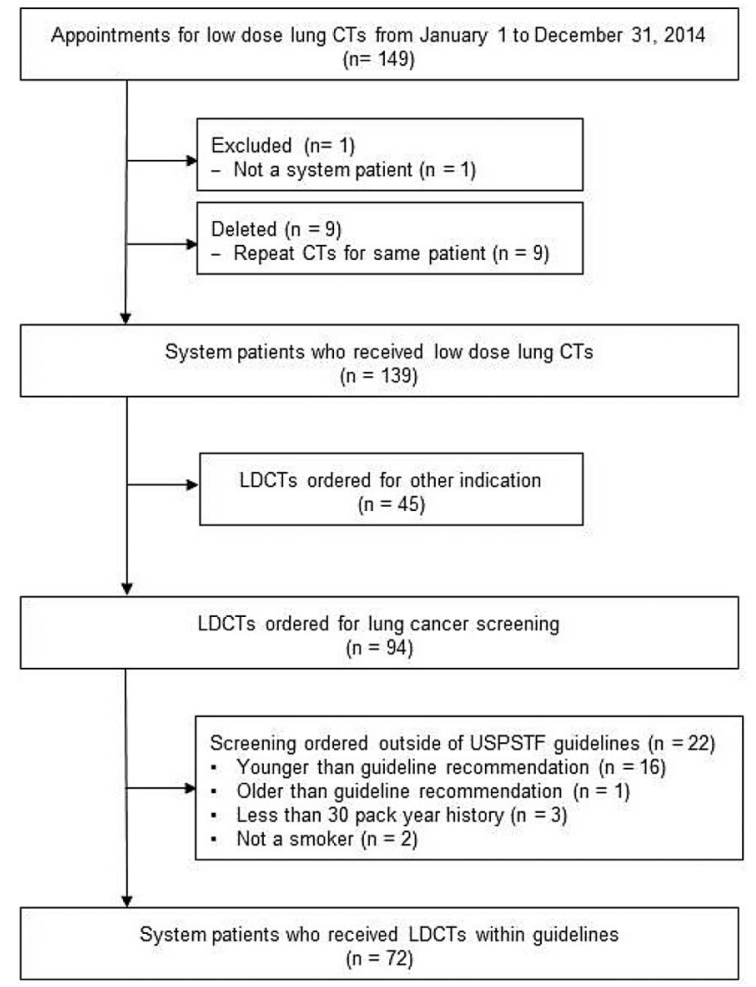

other than nodules, such as emphysema. In this second phase investigators coded radiologic findings as clinically insignificant, clinically significant (other than emphysema), or emphysema.

Descriptive statistics were calculated to quantify the outcome variables. $\chi^{2}$ Statistics tested for significant differences defined as $P<.05$.

\section{Results}

From January to December 2014, 149 patients received LDCT scans in the radiology department. Of these, 94 scans were ordered specifically as screening tests. The final chart review occurred in February 2015. Among the screening tests, $21(22.3 \%)$ were ordered outside of guidelines. Providers ordered LDCT screening outside of age guidelines-both younger $(\mathrm{n}=15)$ and older $(\mathrm{n}=$ 1) than recommended-and for patients who did not meet the smoking behavior guidelines, with either too little smoking history $(\mathrm{n}=3)$ or no smoking history at all $(\mathrm{n}=2)$.

Seventy-two cases met USPSTF criteria to receive LDCT screening. Of these, 19 (26.4\%) were 
female and $53(73.6 \%)$ were male. The mean age was 62.48 years (standard deviation, 5.93 years; range, 55-79 years). LDCT was ordered by primary care providers as designated by the patientcentered medical home (54.2\%), other primary care providers (19.4\%), pulmonologists (20.8\%), and oncologists $(5.6 \%)$.

Table 1 presents the radiologic findings and the number patients for whom additional imaging studies were ordered as prompted by computed tomography results. Notably, almost half of detected nodules $<4$ $\mathrm{mm}$ prompted additional screening, although this category would not require additional scanning based purely on Fleischner Society criteria. At the time of final coding, 2 patients had received treatment for lung cancer and 1 for breast cancer, all of which were initially detected through LDCT. In the second coding phase, of scans that showed no nodules, 6 cases were categorized as clinically significant (other than emphysema), and 7 were categorized as emphysema.

\section{Discussion}

Nearly a fourth of patients receiving screening LDCT at this community hospital in the first year after the publication of USPSTF recommendations did not actually meet the published screening criteria. Results showed that $65.3 \%$ of LDCT screening scans detected nodules of any size, and among the remaining $34.7 \%$, half had another clinically significant finding. This is a considerably larger proportion of actionable findings than in the NLST, in which $27 \%$ of participants had some abnormality identified on their first screening. ${ }^{3}$ These results highlight an increased diagnostic burden introduced by the guideline. This difference between these findings and those of the

Table 1. Low-Dose Computed Tomography Findings By Fleischner Society Categories

\begin{tabular}{|c|c|c|}
\hline Category & $\begin{array}{c}\text { Patients, } \\
\mathrm{n}(\%)^{*}\end{array}$ & $\begin{array}{c}\text { Prompted additional } \\
\text { imaging, }^{\dagger} \mathrm{n} \text { (\% of } \\
\text { category) }\end{array}$ \\
\hline 0 (No finding) & $25(34.7)$ & $3(12)$ \\
\hline 1 ( $\leq 4-\mathrm{mm}$ nodule $)$ & $32(44.4)$ & $14(43.8)$ \\
\hline $2(>4-$ to $6-\mathrm{mm}$ nodule $)$ & $9(12.5)$ & $6(66.7)$ \\
\hline $3(>6-$ to $8-\mathrm{mm}$ nodule) & $3(4.2)$ & $3(100)$ \\
\hline $4(>8-m m$ nodule $)$ & $3(4.2)$ & $3(100)$ \\
\hline
\end{tabular}

*Of at total of 72 .

${ }^{\dagger} \chi^{2}(4,72)=19.97 ; P<.001$.
NLST could be related to the health status of patients, who may have been generally healthier in the NLST compared with the clinical population studied here. Clinicians must consider a patient's overall health and comorbidities when considering screening. ${ }^{8}$ Almost half of the nodules $<4 \mathrm{~mm}$ resulted in additional imaging studies being done. This is a more aggressive approach than is recommended for incidentally identified nodules.

Published guidelines will never be implemented exactly as written or as intended. After translating the new guideline into practice, the cost-benefit of screening LDCT may require reassessment in clinical decision making and reinforces the need for a shared decision-making model, as emphasized by the Centers for Medicare and Medicaid Services. ${ }^{9}$

\section{Future Research}

Because the USPSTF guideline was still in its first year of implementation during this study, there is need for further investigation of LDCT screening implementation and implications. Additional research on the medical reasons for the practice of expanding guidelines and the resulting outcomes is needed.

\section{Limitations}

Analysis was limited to one community hospital's experience. It is not known how many patients within the hospital's health care system were actually eligible for LDCT, so a rate of adherence to the guidelines cannot be calculated. Results also are limited by the sample size and the single-site nature of the study.

\section{Conclusion}

As screening guidelines emerge and change, providers and patients are faced with uncertainty around how new guidelines will translate into actual clinical practice. This study demonstrates that new lung cancer screening guidelines will not always be implemented as envisioned by the USPSTF, and that the burden of follow-up imaging may be significantly higher than expected. Additional research into the how these guidelines translate into practice is needed to understand better their true impact on health care.

\section{References}

1. US Cancer Statistics Working Group. United States cancer statistics: 1999-2011 incidence and mortality 
Web-based report. Atlanta: Department of Health and Human Services, Centers for Disease Control and Prevention and National Cancer Institute; 2015. Available from: www.cdc.gov/uscs. Accessed March 26, 2015.

2. Moyer VA. Screening for lung cancer: U.S. Preventive Services Task Force recommendation statement. Ann Intern Med 2014;160:330-8.

3. Aberle DR, Adams AM, Berg CD, et al. Reduced lung-cancer mortality with low-dose computed tomographic screening. N Engl J Med 2011;365:395409.

4. Seehusen DA. Should family physicians routinely screen for lung cancer in high-risk populations? No: the USPSTF's recommendation for lung cancer screening is overreaching. Am Fam Physician 2014; 90:73d-4d.

5. Aberle DR, Adams AM, Berg CD, et al. Baseline characteristics of participants in the randomized na- tional lung screening trial. J Natl Cancer Inst 2010; 102:1771-9.

6. Underwood JM, Townsend JS, Tai E, et al. Racial and regional disparities in lung cancer incidence. Cancer 2012;118:1910-8.

7. MacMahon H, Austin JH, Gamsu G, et al; Fleischner Society. Guidelines for management of small pulmonary nodules detected on CT scans: a statement from the Fleischner Society. Radiology 2005; 237:395-400.

8. Schneider D, Arenberg D. Competing mortality in cancer screening: a teachable moment. JAMA Intern Med 2015;175:896-7.

9. Decision memo for screening for lung cancer with low dose computed tomography (LDCT) (CAG00439N). Baltimore: Centers for Medicare \& Medicaid Services; 2015. Available from: https:// www.cms.gov/medicare-coverage-database/details/ nca-decision-memo.aspx? NCAId=274. Accessed November 17, 2015. 